\title{
ÉTUDE GRITIQUE ET AMÉLIORATION DES MÉTHODES DE MESURE DES GONSTANTES ÉLASTO-OPTIQUES PAR LES ULTRA-SONS
}

\author{
Par JAcques DURAN, \\ Laboratoire d'Optique Physique de l'École Supérieure de Physique et Chimie, Paris. \\ (Reçu le 18 décembre 1958.)
}

\begin{abstract}
Résumé. - On étudie les limitations intrinsèques et expérimentales des méthodes de mesure des constantes élasto-optiques par les ultrasons. On examine successivement les méthodes " $B$ » et " $C$ » de Mueller et on propose une explication théorique des anomalies observées par certains auteurs.

On montre que la considération des propriétés de modulation du flux diffracté s'avère plus profitable que l'utilisation des sous-composantes induites par effet Doppler dans les différents ordres de diffraction. Dans cette optique, on propose une nouvelle méthode de mesure permettant de s'affranchir en grande partie du flux parasite et d'éviter l'usage de l'extrapolation à intensité U.S. nulle décrite par Mueller.
\end{abstract}

Abstract. - The intrinsic and experimental limitations of various methods of measurement of elasto-optical constants by ultrasonic waves are investigated with particular emphasis on Mueller's work ("B" and "C"' methods). A theoretical explanation is offered for certain anomalies reported by some authors in a few previous papers.

It is shown that the modulation properties of the diffracted light should be considered rather than the subcomponents induced by means of the Doppler effect in the various diffraction orders. Bearing this in mind, we suggest a new method which greatly lowers the importance of the stray light and avoids the extrapolation to zero ultrasonic intensity.

I. Introduction. - L'étude des constantes élastooptiques et de leur dispersion a connu ces dix dernières années un regain d'intérêt :

- Sur le plan pratique, en vue de l'obtention de modulateurs photoélastiques à hautes performances.

- Sur le plan théorique, par l'utilisation des phénomènes de dispersion pour la détermination des facteurs microscopiques de Mueller (par exemple, les longueurs d'ondes d'inversion de la biréfringence des monocristaux cubiques [2]).

Malheureusement, les mesures des constantes élastooptiques sont souvent entachées d'erreurs supérieures à $5 \%$ et dans de nombreux cas ces incertitudes limitent la portée des conclusions théoriques que l'on pourrait être amené à tirer de leur examen.

Parmi les nombreuses méthodes de mesures proposées, celles utilisant la diffraction par les ultra-sons (méthodes de Mueller) ont été d'une grande utilité conjointement aux mesures à contraintes statiques. Gependant, ces méthədes, appliquées sous des formes diverses, l'ont été presque toujours avec des procédés de détection classiques : visuels ou photographiques.

Nous montrons dans ce qui suit qu'une analyse minutieuse des méthodes de Mueller permet d'expli quer de façon précise les performances relativement médiocres obtenues expérimentalement.

Nous rappelons d'abord leur principe, en utilisant une nouvelle formulation pour le flux diffracté par les ondes U.S. stationnaires dans les solides. Après avoir calculé la sensibilité limite des mesures visuelles dans le cas idéal, nous montrons que le flux parasite diffusé dans l'ordre 1 de diffraction devient rapidement prohibitif, et nous proposons, dans ce sens, une explication des résultats expérimentaux anormaux obtenus par certains auteurs. Nous développons ensuite le principe d'une nouvelle méthode de mesure utilisant le procédé de la détection accordée qui possède, en plus des avantages classiques de ces méthodes (faible bande passante, détection linéaire éventuelle...), l'intérêt d'affranchir en grande partie la mesure du flux parasite et de permettre ainsi d'améliorer très sensiblement la précision des mesures des rapports des constantes élasto-optiques.

II. Rappel des méthodes de mesures des constantes élasto-optiques par les U.S. - Mueller [1] a décrit en 1938 trois méthodes de mesure des rapports des constantes élasto-optiques $P_{i j}$ utilisant les propriétés 

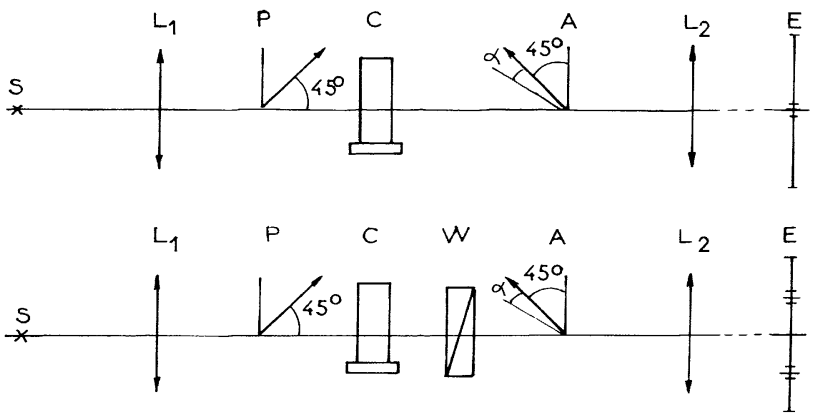

FIG. 1. - Dispositif expérimental pour la méthode $B$ Dispositif expérimental pour la méthode $\mathrm{C}: \mathrm{S}$, fente source ; P, A, Polariseurs ; C, Cristal excité par U.S. ; $\mathrm{L}_{1}, \mathrm{~L}_{2}$, Lentilles; $\mathrm{W}$, Wollaston; E, Spectres de diffraction.

de la lumière diffractée par les U.S. se propageant dans les solides.

Les méthodes les plus utilisées ont été les méthodes dites « $\mathrm{B}$ » (verres et monocristaux cubiques) et « $\mathrm{G}$ » (verres, cristaux cubiques, cristaux uniaxes et biaxes [3]).

La méthode $B$ consiste à mesurer les angles de polarisation des différents ordres de diffraction. On obtient le rapport $R=P_{i j} / P_{i k}$ suivant la relation (à l'ordre 1 de diffraction) :

$$
R=\lim _{v \rightarrow 0} \frac{J_{1}(R v)}{J_{1}(v)}=\lim _{v \rightarrow 0} \operatorname{tg}\left(\alpha+45^{\circ}\right)
$$

dans les cas d'ondes U.S. progressives.

La méthode $\mathrm{G}$ exige la mesure du rapport des intensités diffractées dans les différents ordres pour deux polarisations parallèle et perpendiculaire à la direction de propagation de l'onde acoustique.

On obtient alors (à l'ordre 1 de diffraction) :

$$
R^{2}=\lim _{v \rightarrow 0} \frac{J_{1}^{2}(R v)}{J_{1}^{2}(v)}=\lim _{v \rightarrow 0} \frac{I_{\perp}}{I_{I /}}
$$

En pratique, il apparaît beaucoup plus aisé d'exciter les cristaux, surtout de faibles dimensions, en ondes stationnaires. Les formules (1) et (2) précédentes ont été alors modifiées en prenant en considération les sous-composantes Doppler induites sur la fréquence lumineuse par la modulation du réseau de phase. Par exemple, pour la sous-composante d'ordre $r$, à l'ordre impair de diffraction $m=2 s+1$, on obtient :

$$
\operatorname{tg}\left(\alpha_{m r}+45^{\circ}\right)=\frac{J_{s-r}(R v / 2) J_{s+r+1}(R v / 2)}{J_{s-r}(v / 2) J_{s+r+1}(v / 2)} .
$$

On peut montrer qu'à l'ordre 1 de diffraction la sous-composante d'ordre $r=0$ est prépondérante et que tout se passe comme si la lumière diffractée à l'ordre $m$ était décomposée en une infinité de souscomposantes d'intensités différentes et polarisées suivant des directions fixes dans le temps mais variables d'une sous-composante à l'autre. L'extinction, à l'aide de l'analyseur A de la lumière diffractée (méthode B), est alors rendue impossible par la présence de souscomposantes d'ordre $r \neq 0$; mais on observe un minimum de lumière diffractée, par exemple à l'ordre 1 pour une condition analogue à celle de l'expression (1) lorsque l'intensité U.S. décroît jusqu'à 0 .

Cependant, si cette explication a rendu de grands services, il semble qu'elle soit critiquable au moins pour deux raisons :

- Les procédés de détection classiques utilisés pour de telles mesures ne permettent pas de distinguer les différentes sous-composantes de l'effet Doppler; en tournant l'analyseur $\mathrm{A}$, on observe une variation continue de la lumière diffractée et non pas de discontinuité du plan de polarisation.

- Cette explication masque totalement les propriétés de modulation dans le temps du flux diffracté (que l'on peut mettre facilement en évidence).

A la suite des travaux de Hiedemann et Cook, puis plus récemment de Hargrove, il apparaît que l'on peut donner une formulation légèrement différente des expressions (1) et (2) qui échappe aux critiques précédentes.

D'autre part, nous verrons ci-dessous l'intérêt que l'on peut retirer d'une telle formulation.

Hiedemann et Cook, puis Hargrove de façon plus générale, ont montré que l'intensité diffractée à l'ordre $m$ par un réseau de phase engendré par une onde U.S. stationnaire est de la forme :

$$
I_{m}=I_{0} J_{m}^{2}\left(2 v_{0} \sin \omega^{*} t\right)
$$

où $\omega^{*}$ est la pulsation des ultra-sons.

$I_{m}$ est alors une fonction du temps; les formules (1) et (2) s'écrivent alors :

$$
\begin{aligned}
R(t)=\operatorname{tg}\left(\alpha+45^{\circ}\right) & =\frac{J_{1}\left(2 R v_{0} \sin \omega^{*} t\right)}{J_{1}\left(2 v_{0} \sin \omega^{*} t\right)} \\
R^{2}(t)=\operatorname{tg}^{2}\left(\alpha+45^{\circ}\right) & =\frac{J_{1}^{2}\left(2 R v_{0} \sin \omega^{*} t\right)}{J_{1}^{2}\left(2 v_{0} \sin \omega^{*} t\right)} .
\end{aligned}
$$

Si $v_{0} \neq 0, \alpha$ est alors fonction du temps. Nous avons tracé sur la figure 2 l'allure de la courbe $\alpha(t)$ pour $R=1,5$ calculée pour deux valeurs de $v_{0}$.

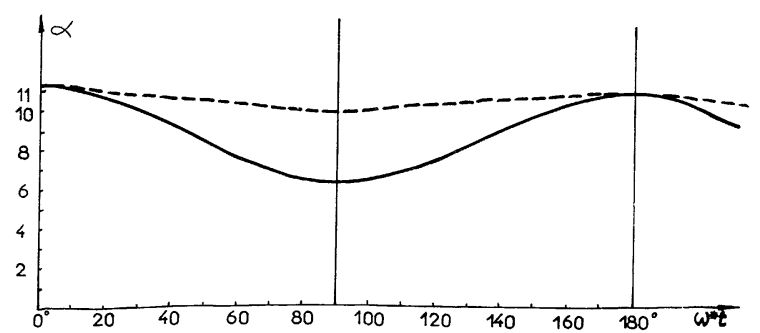

FIG. $2 .-\alpha=f(t)$. Ondes stationnaires : $v=2 v_{0} \sin \omega^{*} t$. $---R=1,5 ; \quad v_{0}=0,25$.

$-R=1,5 ; \quad v_{0}=0,5$. 


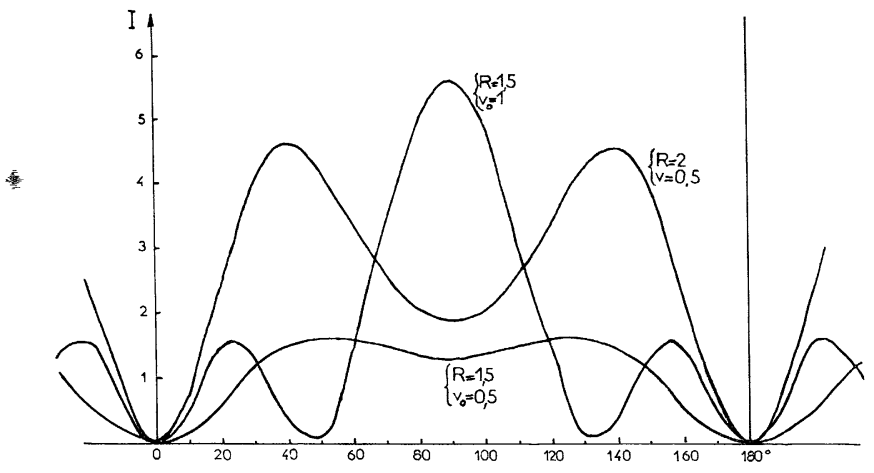

FIG. 3. - Ondes stationnaires :

$I=100\left[J_{1}(R v)-J_{1}(v)\right]^{2} ; \quad v=2 v_{0} \sin \omega^{*} t$.

La figure 3 représente les variations dans le temps du flux diffracté à l'ordre 1 , lorsque $\alpha=0$ (méthode $\mathrm{B}$, polariseur et analyseur croisés), pour différentes valeurs de $R$ et de $v_{0}$.

III. Etude critique des méthodes de Mueller. Expérimentalement, le procédé utilisé consiste à faire décroître progressivement l'intensité d'excitation ultrasonore, à mesurer les valeurs de $R_{-\varepsilon}$ correspondantes, puis à extrapoler à intensité U.S. nulle. On obtient alors des courbes du type de celle tracée sur la figure $4 \mathrm{a}$.

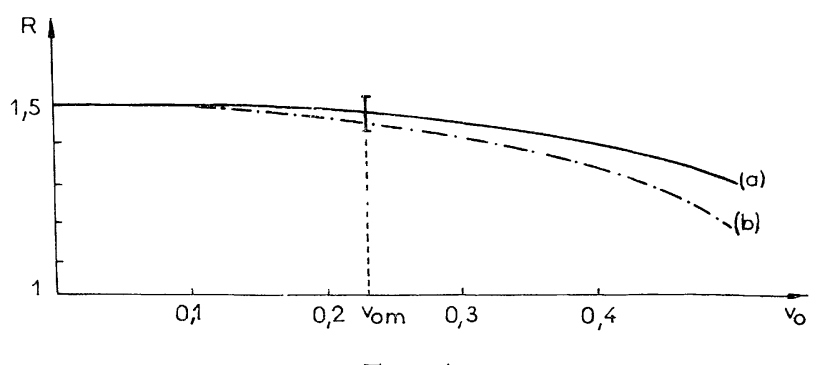

FIG. 4.

De nombreux auteurs [1], [3], [5] ont rapporté des courbes de ce type, tracées expérimentalement suivant la méthode $B$.

Il apparaît que la précision sur la mesure de $R$ dépend de deux facteurs :

- la plus petite valeur de $v_{0}$ utilisable pour la mesure,

- l'erreur faite sur $R_{-\varepsilon}$ avec cette valeur de $v_{0}$.

Nous allons examiner successivement, dans le cas des détections de type classique (« en moyenne »), les précisions limites obtenues avec des montages supposés sans flux parasite, puis nous montrerons l'importance considérable du flux parasite sur les mesures. Dans le premier cas, il nous faudra distinguer le type de détection utilisée; dans le second cas, nous vcrrons que la précision limite, dans des conditions convenables, n’en dépend pas.
A. Sensibilité limite des méthodes a DÉtection Visuelle en l'ABSEnce de flux parasite. - Dans le cas de la méthode $\mathrm{C}$, par exemple, il s'agit de comparer les brillances des deux images de la fente source séparées par le Wollaston.

Si $E_{0}$ est l'éclairement des deux images de la source, l'œil perçoit alors $k_{1} E_{0}$ et $k_{2} E_{0}$ à l'ordre 1 de diffraction. Or :

$$
\begin{aligned}
& k_{1}=\frac{1}{T} \int_{0}^{T} J_{1}^{2}\left(2 R v_{0} \sin \omega^{*} t\right) \\
& k_{2}=\frac{1}{T} \int_{0}^{T} J_{1}^{2}\left(2 v_{0} \sin \omega^{*} t\right) .
\end{aligned}
$$

L'analyseur A sera tourné, d'après (4), de façon à conduire à l'égalité des brillances des deux franges homologues (fig. 1, méthode $\mathrm{G}$ ), d'un angle $\alpha$ tel que :

$$
\operatorname{tg}^{2}\left(\alpha+45^{\circ}\right)=\frac{k_{1}}{k_{2}}=R_{-\varepsilon}^{2}
$$

et une différence de brillance $\Delta E$ conduit à une erreur sur $R_{-\varepsilon}^{2}$ :

$$
\frac{\Delta R_{-\varepsilon}^{2}}{R_{-\varepsilon}^{2}}=\frac{\Delta E}{E_{0}} \cdot \frac{k_{1}+k_{2}}{k_{1} k_{2}} .
$$

Si l'on suppose que l'on se trouve dans les conditions idéales de l'analyseur à pénombre, d'après Bruhat [7], l'œil ne peut apprécier des différences de brillances $\Delta E$ telles que :

$$
\frac{\Delta E}{E_{0}} \cdot \frac{k_{1}+k_{2}}{k_{1} k_{2}}<1 / 50
$$

ce qui donne une erreur relative sur $R_{-\varepsilon}^{2}$ :

$$
\frac{\Delta R_{-\varepsilon}^{2}}{R_{-\varepsilon}^{2}} \# 2 \times 10^{-2} \text {. }
$$

Ces conditions idéales seront réalisées pour l'éclairement minimum des deux franges, compte tenu du fait que la perception visuelle impose que :

$$
\frac{k_{1} k_{2}}{k_{1}+k_{2}} \geqslant\left(\frac{1}{57}\right)^{2} \quad \text { (lampe } \mathrm{Hg} 80 \text {, raie verte). }
$$

La plus petite valeur de $k_{2}$ utilisable est donc :

$$
k_{2 m} \# 5 \cdot 10^{-4} \text { pour } R=1,5
$$

et pour $v_{0 m}$ minimum, sachant que si $v_{0}$ est petit :

$$
\begin{gathered}
\frac{1}{T} \int_{0}^{T} J_{1}^{2}\left(2 v_{0} \sin \omega^{*} t\right) \mathrm{d} t \not \frac{1}{2} v_{0}^{2} \\
v_{0 m} \# 3,1 \times 10^{-2} .
\end{gathered}
$$

Une telle valeur de $v_{0 m}$ donnerait une valeur de $R$ exacte à mieux que $10^{-3}$ près, ce qui permettrait de faire cette mesure sans extrapolation. La précision sur $R$ serait alors, en tenant compte de l'erreur donnée en (6), meilleure que $2 \%$.

B. Sensibilité limite des méthodes a Détection PHOTOÉlectrique EN L'ABSENCE DE FluX PARASITE. Si l'on suppose que le dispositif (par exemple électronique) de repérage de l'égalité des flux diffractés ne 
limite pas la précision de la mesure par lui-même, il est facile de montrer que l'on pourrait améliorer considérablement la précision de la mesure de $R$ en utilisant un PM à faible courant d'obscurité.

Cependant, les conditions précédentes (analyseur à pénombre pour détection visuelle) sont loin d'être réalisées dans un montage d'étude des constantes élasto-optiques. En effet :

- Les raies comparées sont très fines et il est difficile de superposer les deux spectres de façon très étroite.

- Les raies du spectre de diffraction à l'ordre 1 contiennent toujours de la lumière parasite issue de l'ordre 0 (ce qui est inhérent au principe de la méthode). Elles contiennent en outre de la lumière dépolarisée ou elliptique provenant de l'optique et des particules diffusantes rencontrées tout le long des rayons lumineux.

Une étude systématique du montage nous a montré qu'avec des conditions rigoureuses (doubles fentes, diaphragme convenable...), il était difficile de descendre, avec une lentille $\mathrm{L}_{2}$ de focale $1,50 \mathrm{~m}$, audessous d'un taux de lumière parasite inférieur à :

$$
\begin{array}{llll}
0,5 \% & \text { pour une fréquence } & \text { U.S. de } & 5 \mathrm{MHz} \\
0,2 \% & - & - & 10 \mathrm{MHz} \\
0,14 \% & - & - & 20 \mathrm{MHz} .
\end{array}
$$

C. Précision des mesures en présence de flux PARASITE. - Nous allons montrer que la présence de flux parasite affecte la mesure de $R_{-\varepsilon}^{2}$ d'une erreur systématique rendant illusoire l'emploi de détecteurs très sensibles.

Soit $\tau E_{0}$ l'éclairement parasite diffusé dans les deux spectres homologues en l'absence de l'analyseur A (méthode $\mathrm{C}$ ).

Si l'égalité de brillance des plages homologues est réalisée :

$\left(k_{1}+\tau\right) \cos ^{2}\left(\alpha+45^{\circ}\right)=\left(k_{2}+\tau\right) \sin ^{2}\left(\alpha+45^{\circ}\right)$.

Si on mesure $\frac{k_{1}+\tau}{k_{2}+\tau}$ au lieu de $k_{1} / k_{2}$, on commet

$$
\frac{\Delta\left(R_{-\varepsilon}^{2}\right)}{R_{-\varepsilon}^{2}}=\frac{\tau}{k_{2}+\tau}\left[\frac{1-R_{-\varepsilon}^{2}}{R_{-\varepsilon}^{2}}\right] .
$$

Si $k_{2} \leqslant \tau$, la mesure est évidemment absurde. Si $k_{2}>\tau$, il vient :

$$
\frac{\Delta R_{-\varepsilon}^{2}}{R_{-\varepsilon}^{2}} \# \frac{\tau}{k_{2}} \cdot \frac{\left[1-R^{2}\right]}{R^{2}} .
$$

On en déduit que si $\tau=0,005$ et $R=1,5$ :

$$
\frac{\Delta R_{-\varepsilon}^{2}}{R_{-\varepsilon}^{2}} \# 0,56 \times \frac{\tau}{k_{2}} \text {. }
$$

- La mesure de $R^{2}$ à $1 \%$ près est illusoire car il faudrait prendre $k_{2} \geqslant 0,28$, ce qui conduirait à une valeur de $v_{0}$ trop grande $\left(v_{0}\right.$ de l'ordre de 0,7$)$.

- La mesure de $R_{-\varepsilon}^{2}$ à $10 \%$ près est possible à condition de prendre $k_{2} \geqslant 0,027$, c'est-à-dire $v_{0} \geqslant 0,23$.

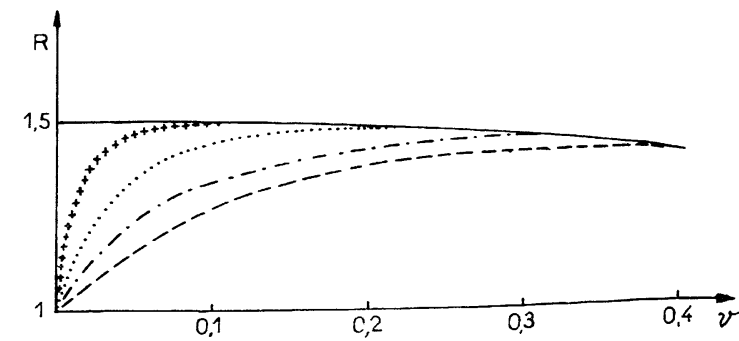

FIG. 5. $-\tau \tau=0 ;-.--\tau=5^{0}{ }_{1}^{\circ}$ oo $;+++\tau=$ $2 \times 10^{-4}$ (mét. B) ; $\cdots \tau=1{ }^{\circ}{ }_{\text {oo }}^{\prime} ;--\tau=1{ }_{\circ}^{\circ}$. Détection en moyenne avec flux parasite.

Nous avons reporté sur la figure 5 les allures des courbes calculées pour différents taux de lumière parasite.

Il est intéressant de comparer ces résultats avec les courbes observées expérimentalement par Gates et Hiedemann [5]. L'analogie des courbes 5 et 6 permet

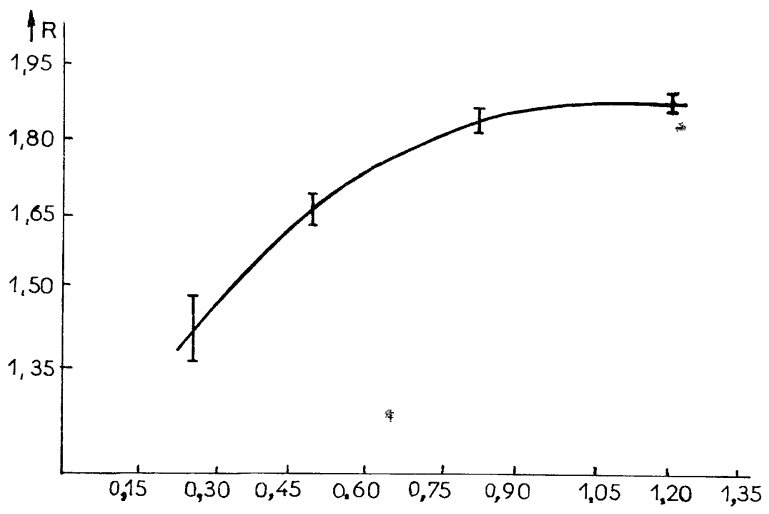

FIG. 6. - Courant dans le transducteur en (ampères) ${ }^{2}$. Verre BSC-I (d'après H. F. Gates et E. A. Hiedemann).

de suggérer une explication, basée sur l'existence du flux parasite, de la décroissance anormale de $R$ lorsque $v_{0} \rightarrow 0$. Dans cette hypothèse, les mesures de L. Bergmann [8], selon lesquelles le rapport $k_{1} / k_{2}$ semblerait plus proche de $R$ que de $R^{2}$ pour les faibles intensités U.S., trouveraient une justification et proviendraient donc d'une limitation expérimentale de la méthode $\mathrm{C}$.

On peut aussi, toujours conformément aux résultats expérimentaux de Gates et Hiedemann, montrer que la méthode $\mathrm{B}$ conduit, dans les cas où elle est utilisable, à des résultats plus précis. En effet, le flux parasite $\tau$ issu en majeure partie de l'ordre 0 devient alors :

$$
\begin{gathered}
\tau^{\prime}=\tau \sin ^{2} \alpha \# 0,036 \tau \text { pour } \quad R=1,5 \\
\text { et : } \quad \tau^{\prime} \# 2 \times 10^{-4} \text { si } \tau=0,005 .
\end{gathered}
$$

On obtient la courbe représentée sur la figure 5 qui ne présente une variation de pente notable qu'en deçà 
de la limite inférieure des valeurs de $v_{0}$ atteintes par ces différents auteurs. On peut observer d'autre part qu'un accroissement de la sensibilité des détecteurs utilisés (remplacement de l'œil par un photomultiplicateur, par exemple) devient illusoire pour les deux méthodes (B et $\mathrm{C}$ ).

IV. Méthode proposée. Détection accordée. - Afin de profiter des qualités du détecteur utilisé, il est important d'essayer de s'affranchir du flux parasite. On propose d'utiliser pour cela les propriétés de modulation dans le temps du flux diffracté. En effet, il apparaît que lorsque l'intensité U.S. décroît vers 0 , l'ordre 1 de diffraction présente une modulation prépondérante suivant l'harmonique $2 \omega^{*}$, tandis que le flux parasite voit son taux de modulation décroître. En développant en série les fonctions de Bessel, pour l'ordre 0 et l'ordre 1 de diffraction, on obtient :

$$
\left\{\begin{array}{c}
I_{0}=I J_{0}^{2}\left(2 v_{0} \sin \omega^{*} i\right)=1-2 X^{2}+3 / 2 X^{4} \ldots \\
I_{1}=I J_{1}^{2}\left(2 v_{0} \sin \omega^{*} t\right)=X^{2}\left(1-X^{2}+5 / 12 X^{4} \ldots\right) \\
X=v_{0} \sin \omega^{*} t
\end{array}\right.
$$

L'amplification sélective sur l'harmonique 2 ne conserve que les termes :

$$
\left\{\begin{array}{l}
I_{02 \omega^{*}}=I \frac{\cos 2 \omega^{*} t}{2}\left(2 v_{0}^{2}-3 / 2 v_{0}^{4} \ldots\right) \\
I_{12 \omega^{*}}=-I \frac{\cos 2 \omega^{*} t}{2}\left(v_{0}^{2}-v_{0}^{4}+\ldots\right) .
\end{array}\right.
$$

$\mathrm{Si}$ on considère que tout le flux parasite est issu de l'ordre 0 de diffraction, l'amplification sélective ne détecte que :

$$
\tau_{2 \omega^{*}}=v_{0}^{2} \tau
$$

$\tau_{2 \omega^{*}}$ décroît très vite lorsque $v_{0} \rightarrow 0$ (comme $v_{0}^{2}$ ), alors que dans les méthodes précédentes le flux parasite était pratiquement constant. Par exemple, pour $\tau=5 \times 10^{-3}$ à $v_{0}=0,01$, on aura $\tau_{2 \omega^{*}}=5 \times 10^{-7}$.

Il faut remarquer cependant que le filtrage sur l'harmonique $2 \mathrm{du}$ signal recueilli dans l'ordre $1 \mathrm{de}$ diffraction ne conduit pas exactement à la même courbe $R(v)$ que dans le cas de la détection en moyenne. Nous avons représenté la courbe théorique obtenue après amplification sélective sur la figure 4 (courbe b). On peut montrer que les courbes a et b de la figure 4 (détection en moyenne et détection accordée) possèdent la même allure autour du point $v=0$.

A. Erreur systématique sur la mesure de $R$ en DÉTECTION AGCORDÉE. - Si nous reprenons l'expression (8) donnant l'erreur systématique commise sur la mesure de $R_{-\varepsilon}^{2}$, nous obtenons :

$$
\frac{\Delta R_{-\varepsilon}^{2}}{R_{-\varepsilon}^{2}} \# \frac{v_{0}^{2} \tau}{k_{2}}\left[\frac{1-R^{2}}{R^{2}}\right] .
$$

Si $v_{0}$ est petit :

$$
k_{2} \# \frac{1}{2} v_{0}^{2}
$$

et alors :

$$
\frac{\Delta R_{-\varepsilon}^{2}}{R_{-\varepsilon}^{2}} \# 2 \tau \frac{1-R^{2}}{R^{2}}
$$

l'erreur systématique est alors constante quelle que soit l'intensité ultrasonore appliquée, tandis qu'elle augmente comme $\left(1 / v_{0}\right)^{2}$ pour les mesures à détection en moyenne. Cette erreur, dont le sens est connu, peut être évaluée. Elle est de l'ordre de grandeur de $\tau$ si $R=1,5$.

Dans ces conditions, il devient possible d'atteindre la sensibilité limite du détecteur puisque le signal et le flux parasite modulés décroissent de la même manière lorsque $v_{0} \rightarrow 0$.

B. Sensibilité limite des mesures a DÉtegtion PHOtó́leGtrique AGGordéE. - Si $I$ est le courant débité par le photomultiplicateur, on peut écrire pour la polarisation afférente à $k_{2}$ :

$$
I=\left(\tau_{\mathrm{c}}+\tau_{2 \omega^{*}}\right) I_{0}+k_{2} I_{0}
$$

où $\tau_{\mathrm{c}}$ est le taux de lumière parasite contenu dans le premier ordre de diffraction et $\tau_{2 \omega^{*}}$ la proportion de lumière modulée issue de l'ordre 0 .

Pour les valeurs de $v_{0}$ qui nous intéressent :

$$
\tau_{\mathrm{c}} I_{0} \gg k_{2} I_{0} \gg \tau_{2 \omega^{*}} I_{0}
$$

Le plus petit signal décelable sera alors égal au bruit Schottky du photomultiplicateur et vaudra :

$$
k_{2 m}=\sqrt{2 e M . \tau_{\mathrm{c}} \Delta f / I_{0}} .
$$

Par exemple, dans les conditions expérimentales suivantes :

$$
2 e M=1,28 \times 10^{-13} \mathrm{MKSA}
$$

( $M$ pouvoir multiplicateur du $\mathrm{PM}$ )

$$
I_{0}=10^{-2} \mathrm{~A}
$$

(lampe SP 500, raie verte)

$$
\begin{gathered}
\tau_{\mathrm{c}}=0,005 \\
\Delta f=1 \mathrm{~Hz}
\end{gathered}
$$

(bande passante du galvanomètre de mesure)

et si l'on suppose toujours que les procédés de comparaison des flux (méthode $\mathrm{C}$ ) ou de détection du minimum de flux (méthode B) n'affectent pas, eux-mêmes, la précision des mesures, on peut alors espérer mesu- 
rer $R_{-\varepsilon}$ à $2,5 \times 10^{-3}$ près pour $v_{0}=10^{-2}$ et avec une approximation de $R$ exacte, bien meilleure que $10^{-3}$. L'extrapolation devient alors superflue et la mesure peut être faite directement avec une précision plus de dix fois supérieure à celle des méthodes précédentes.

\section{BIBLIOGRAPHIE}

[1] Muel.ler (H.), Z. Kristall., Band 99, 1938, Leipzig.

[2] Rahman (A.) et Iyengar (K. S.), Physics Letters (Netherland), 1967, 25 A, 6, 478.

[3] Narasimmamurthy (T. S.), Acta Cryst., 1950, 14, 1176.

[4] Hargrove (T. E.), J. Acoust. Soc. Amer., 1967, 41, $1,91$.
[5] Gates (H. F.) et Hiedemann (G. A.), J. Acoust. Soc. Amer., 1956, 28, 6.

[6] Cook et Hiedemann (G. A.), J. Acoust. Soc. Amer., 1961, 33, 7.

[7] BRUhAT, Traité de polarimétrie, 1930, p. 70.

[8] Bergmann (L.), Der Ultraschall, 6e éd., 1954, p. 663. 